\title{
Brucellosis: a retrospective evaluation of 164 cases
}

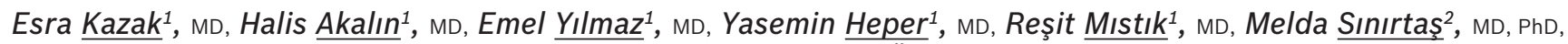 \\ Cüneyt Özakın $^{2}, \mathrm{MD}$, Güher $\underline{\mathrm{Göral}}^{2}$, PhD, Safiye $\underline{\mathrm{Helvacl}}^{1}, \mathrm{MD}$
}

INTRODUCTION Brucellosis is a public health problem that is prevalent in several developing countries. METHODS The clinical and laboratory characteristics of 164 cases of brucellosis in Bursa, Turkey, were retrospectively evaluated.

RESULTS The ages of the 164 patients ranged from 15-85 years. All of the patients underwent the Rose Bengal test and $163(99.4 \%)$ patients tested positive. $122(74.4 \%)$ patients were diagnosed with acute brucellosis, $31(18.9 \%)$ with subacute brucellosis and $11(6.7 \%)$ with chronic brucellosis. Focal involvement was found in $101(61.6 \%)$ patients. Although patients with focal involvement had a higher white blood cell count $(p=0.002)$, those without focal involvement had higher aspartate transaminase and alanine transaminase values, and lower platelet values $(p=0.005,0.007$ and 0.039 , respectively). Spondylodiscitis was observed on imaging in $58(66.7 \%)$ of the 87 patients who presented with back pain. Among the 118 patients who were examined within the first month of treatment, $79(66.9 \%)$ responded to treatment. The relapse rate was $11.6 \%$ among all 164 patients.

CONCLUSION Brucellosis should be considered as a differential diagnosis among patients who present with fever, and joint or back pain. Focal involvement should be investigated in the presence of leucocytosis, and subacute or chronic forms of brucellosis. To identify cases of spondylodiscitis, radiography should be performed in patients who present with back pain.

Keywords: brucellosis, diagnosis, epidemiology, musculoskeletal, spondylodiscitis

\section{INTRODUCTION}

Brucellosis is a disease that commonly afflicts herbivores and can result in abortions as well as the loss of milk and meat production in animals. This disease is generally transmitted to humans via infected tissue, body fluids, urine, milk or milk products. The agent of brucellosis is a small, Gram-negative, unencapsulated and nonsporulating coccobacilli from the Brucella genus. This genus is divided into six major nomen species based on natural host preference, and cultural, metabolic and antigenic characteristics. The typical reservoirs of Brucella melitensis (B. melitensis) are goats and sheep, while Brucella abortus, Brucella suis and Brucella canis (B. canis) are found principally in cattle, swine and canines, respectively; $B$. canis is an infrequent cause of human infection. ${ }^{(1)}$ $B$. melitensis accounts for the majority of the human brucellosis cases in Turkey and throughout the world. ${ }^{(2}$

The symptoms of brucellosis are generally nonspecific and include high fever, night sweats and joint pain. The clinical and laboratory features of brucellosis tend to vary, as it is a systemic infection that can involve any organ of the body. Diagnosing brucellosis is difficult due to the nonspecific signs and symptoms of the disease. According to the duration of the symptoms, brucellosis can be categorised as acute, subacute or chronic. Chronic brucellosis is usually caused by a persistent foci of infection in tissue. ${ }^{(1)}$

While brucellosis is observed all over the world, it is most common in Mediterranean countries, the Arabian Peninsula, India, and Central and South America. As brucellosis is usually transmitted through the consumption of unpasteurised milk and dairy products, it is encountered as an 'occupational disease' in countries where pasteurised dairy products are consumed; this is especially the case if the infection is found among veterinarians, slaughterhouse workers, farmers and laboratory workers. ${ }^{(1)}$ Brucellosis is frequently observed in Turkey. The disease is significant due to its high morbidity and capacity to incur labour and economic losses (due to its chronic and recurring nature) in endemic areas. In Turkey, brucellosis most commonly involves the osteoarticular and haematopoietic systems. ${ }^{(3)}$ We herein report the findings of our retrospective review of the epidemiological, clinical and laboratory characteristics of 164 cases of brucellosis that were seen in the Infectious Diseases Clinic, Faculty of Medicine, Uludag University, Bursa, Turkey.

\section{METHODS}

In the present study, the case files of 164 immunocompetent brucellosis patients (77 [47.0\%] female and 87 [53.0\%] male) who presented to the Infectious Diseases Clinic, Faculty of Medicine, Uludag University, Bursa, Turkey, between 1986 and 2012 were retrospectively evaluated. Patients who met the following diagnostic criteria for brucellosis were included: (a) the diagnosis was accompanied by clinical findings; (b) there was positive growth of Brucella species in the blood culture or any other body fluid/tissue cultures; or (c) in the absence of bacteriological confirmation, identification of positivity in a single serum sample titre of 1:160 and above in the serum agglutination test (SAT) and/or a minimum fourfold increase identified per titre within a 2-3 week interval. ${ }^{(1)}$ For blood and body fluid cultures, conventional brain-heart infusion

${ }^{1}$ Infectious Diseases and Clinical Microbiology Department, ${ }^{2}$ Medical Microbiology Department, Uludag University Medical School, Bursa, Turkey Correspondence: A/Prof Esra Kazak, Consultant, Infectious Diseases and Clinical Microbiology Department, Faculty of Medicine, Uludag University, Bursa, Turkey. eskazak@hotmail.com 
biphasic growth medium with prolonged incubation was used in the years 1986-1992, while BACTEC MR730 was used from 1993-1995 and the BACTEC ${ }^{\text {TM }} 9000$ (9120-9240) Phoenix (BD Diagnostics, Sparks, MD, USA) system was used from 1995-2012. ${ }^{(4)}$

Each patient's signs and symptoms, laboratory values, physical examination results, and radiological signs were noted from electronic patient charts. Patients without malignancy who did not receive immunosuppressive or steroid therapy were accepted as immunocompetent. The patients were divided into three groups based on the duration of their symptoms: acute (0-2 months), subacute (3-12 months) and chronic (> 12 months). Although brucellosis is a systemic infection that can involve any organ or system, when specific organ involvement was dominant in any of the 164 patients, the situation was defined as 'focal' or 'localised'. (1) The patients were primarily provided with doxycycline-rifampicin and doxycycline-streptomycin treatments, according to World Health Organization (WHO) guidelines. ${ }^{(5)}$ Tetracycline and streptomycin treatment was provided if the patients were treated before the publication of the current WHO guideline. In cases of meningitis, ceftriaxone was added to the treatment within the first 30 days, while ciprofloxacin was added during the treatment period for patients with spondylodiscitis. Due to side effects and some cases of patient intolerance, varying combinations of doxycycline, rifampicin, ceftriaxone, streptomycin, ciprofloxacin and trimethoprim-sulfamethoxazole treatments were used; the treatments were tailored according to the age, clinical status, focal involvement and laboratory data of each patient. ${ }^{(1)}$ Treatment failure was defined as continuation of the initial signs and symptoms in the second month of treatment, while relapse was defined as recurrence of clinical signs and symptoms after cessation of treatment in patients who had responded to treatment. ${ }^{(6,7)}$ Patients were deemed to have responded to treatment if they had lower body temperatures, and no longer had any signs or symptoms within the first month of treatment. ${ }^{(6)}$

Shapiro-Wilk test was used to check the normality of the data. If the variables were normally distributed, the data was presented as mean \pm standard deviation. If the variables were not normally distributed, the data was presented as median (range). Mann-Whitney $U$ test was used to compare the patients with and without focal involvement, and Pearson chi-square test was used for categorical data analysis. A p-value $<0.05$ indicated statistical significance.

\section{RESULTS}

The 164 patients included in the present study were aged 15-85 years. Their occupations are shown in Table I. Among the 164 patients, $109(66.5 \%)$ resided in urban areas and $55(33.5 \%)$ in rural areas. Evaluation of patient histories revealed that $83(50.6 \%)$ patients consumed unpasteurised cheese, $61(37.2 \%)$ had animal contact and $30(18.3 \%)$ both consumed unpasteurised cheese and had animal contact. The transmission route in 48 (29.3\%) patients was unknown. In one patient, sexual transmission was suggested, as she lacked a history of high-risk
Table I. Occupations of patients with brucellosis $(n=164)$.

\begin{tabular}{lc}
\hline Occupation & No. (\%) \\
\hline Housewife & $53(32.3)$ \\
Animal husbandman & $46(28.0)$ \\
Veterinarian & $14(8.5)$ \\
Laboratory personnel & $3(1.8)$ \\
Cook & $1(0.6)$ \\
Others* & $47(28.7)$ \\
\hline
\end{tabular}

*Including government clerks, students, etc.

animal contact and consumption of unpasteurised dairy products, but had contracted brucellosis one month after her spouse (who worked at a slaughterhouse) suffered from brucellosis. Laboratory transmission occurred in $1(0.6 \%)$ patient. $4(2.4 \%)$ patients had a family history of recent brucellosis.

The duration of patients' symptoms prior to clinical evaluation ranged from seven days to three years. Among the 164 patients, $122(74.4 \%), 31(18.9 \%)$ and $11(6.7 \%)$ patients were diagnosed with acute, subacute and chronic brucellosis, respectively. The complaints of the patients at the time of admission to our department are shown in Table II, while the findings of the physical examinations conducted at the time of admission are presented in Table III. Fever was the most common finding.

Focal involvement was found in 101 (61.6\%) of the 164 patients. It was identified in $52.5 \%$ of the patients with acute brucellosis, $93.5 \%$ of the patients with subacute brucellosis and $72.7 \%$ of the patients with chronic brucellosis. Among the 101 patients with focal involvement, the proportions of the patients with acute, subacute and chronic brucellosis were $64(63.4 \%), 29(28.7 \%)$ and $8(7.9 \%)$, respectively. The location and distribution of the focal involvement identified in the patients are shown in Table IV.

Spondylodiscitis was identified on imaging in $58(66.7 \%)$ of the 87 patients who presented with back pain, and in $6(7.8 \%)$ of the 77 patients who did not present with back pain $(p<0.001)$. The laboratory test results of the patients at the time of diagnosis (i.e. before antibiotic treatment) are shown in Table V. All of the patients tested negative for hepatitis $B$ virus surface antigen and anti-hepatitis $C$ virus; none of them had a history of exposure to hepatotoxic agents. A comparison of the groups with and without focal involvement showed that the differences were statistically significant with regard to white blood cell count, aspartate transaminase (AST) level, alanine transaminase (ALT) level and platelet count, and not statistically significant with regard to haemoglobin level, sedimentation rate and C-reactive protein level. Although white blood cell counts were higher among the patients with focal involvement, AST and ALT values were higher among the patients without focal involvement, who also had lower platelet counts (Table VI).

All 164 patients underwent the Rose Bengal test and the results were positive in 163 (99.4\%) patients. The Brucella species was detected in the blood culture of the patient who tested negative for brucellosis in the Rose Bengal test and SAT. Of the 114 patients for whom blood samples were obtained at admission, the blood cultures of 68 (59.6\%) patients tested positive for the Brucella 
Table II. Complaints at the time of admission.

\begin{tabular}{|c|c|c|c|c|}
\hline \multirow[t]{2}{*}{ Complaint } & \multicolumn{4}{|c|}{ No. (\%) } \\
\hline & $\begin{array}{c}\text { Total } \\
(\mathrm{n}=164)\end{array}$ & $\begin{array}{l}\text { Acute brucellosis } \\
\qquad(n=122)\end{array}$ & $\begin{array}{l}\text { Subacute brucellosis } \\
\qquad(n=31)\end{array}$ & $\begin{array}{l}\text { Chronic brucellosis } \\
\qquad(n=11)\end{array}$ \\
\hline Fever & $123(75.0)$ & $104(85.2)$ & $16(51.6)$ & $3(27.3)$ \\
\hline Malaise & 114 (69.5) & $91(74.6)$ & $20(64.5)$ & $3(27.3)$ \\
\hline Decreased appetite & $84(51.2)$ & $75(61.5)$ & $6(19.4)$ & $3(27.3)$ \\
\hline Arthralgia & $90(54.9)$ & $65(53.3)$ & $20(64.5)$ & $5(45.5)$ \\
\hline Back pain & $87(53.0)$ & $53(43.4)$ & $26(83.9)$ & $8(72.7)$ \\
\hline Weight loss & $38(23.2)$ & $35(28.7)$ & $2(6.5)$ & $1(9.1)$ \\
\hline Headache & 31 (18.9) & $26(21.3)$ & $3(9.7)$ & $2(18.2)$ \\
\hline Myalgia & $15(9.1)$ & $12(9.8)$ & $3(9.7)$ & 0 \\
\hline Depression & $6(3.7)$ & $3(2.5)$ & 0 & $3(27.3)$ \\
\hline
\end{tabular}

Table III. Findings of the physical examinations at the time of admission.

\begin{tabular}{|c|c|c|c|c|}
\hline \multirow[t]{2}{*}{ Finding } & \multicolumn{4}{|c|}{ No. (\%) } \\
\hline & $\begin{array}{c}\text { Total } \\
(n=164)\end{array}$ & $\begin{array}{l}\text { Acute brucellosis } \\
\qquad(n=122)\end{array}$ & $\begin{array}{l}\text { Subacute brucellosis } \\
\qquad(n=31)\end{array}$ & $\begin{array}{l}\text { Chronic brucellosis } \\
\qquad(n=11)\end{array}$ \\
\hline Lymphadenopathy & $16(9.8)$ & $14(11.5)$ & $1(3.2)$ & $1(9.1)$ \\
\hline Skin involvement & $7(4.3)$ & $6(4.9)$ & $1(3.2)$ & 0 \\
\hline Hepatomegaly & $35(21.3)$ & $32(26.2)$ & $2(6.5)$ & $1(9.1)$ \\
\hline Splenomegaly & $31(18.9)$ & $31(25.4)$ & 0 & 0 \\
\hline Meningeal irritation signs & $9(5.5)$ & $8(6.6)$ & $1(3.2)$ & 0 \\
\hline Orchitis* & $7(8.0)$ & $5(7.7)$ & $1(5.8)$ & $1(20.0)$ \\
\hline Cardiac murmur & $5(3.0)$ & $4(3.3)$ & 0 & $1(9.1)$ \\
\hline Arthritis & $11(6.7)$ & $7(5.7)$ & $4(12.9)$ & 0 \\
\hline
\end{tabular}

*Percentage calculated based on no. of male patients in each category.

Table IV. Location and distribution of the focal involvement identified in the patients with brucellosis.

\begin{tabular}{|c|c|c|c|c|}
\hline \multirow[t]{2}{*}{ Variable } & \multicolumn{4}{|c|}{ No. (\%) } \\
\hline & $\begin{array}{c}\text { Total } \\
(n=164)\end{array}$ & $\begin{array}{l}\text { Acute brucellosis } \\
\qquad(n=122)\end{array}$ & $\begin{array}{l}\text { Subacute brucellosis } \\
\qquad(n=31)\end{array}$ & $\begin{array}{c}\text { Chronic brucellosis } \\
(\mathbf{n}=11)\end{array}$ \\
\hline Peripheral arthritis & $11(6.7)$ & $7(5.7)$ & $4(12.9)$ & 0 \\
\hline Knee & $8(4.9)$ & $4(3.3)$ & $4(12.9)$ & 0 \\
\hline Hip & $1(0.6)$ & $1(0.8)$ & 0 & 0 \\
\hline Spondylodiscitis & $64(39.0)$ & $30(24.6)$ & $26(83.9)$ & $8(72.7)$ \\
\hline Sacroiliitis & $33(20.1)$ & $25(20.5)$ & $7(22.6)$ & $1(9.1)$ \\
\hline Bursitis & $3(1.8)$ & $2(1.6)$ & $1(3.2)$ & 0 \\
\hline Skin involvement & $7(4.3)$ & $6(4.9)$ & $1(3.2)$ & 0 \\
\hline Neurobrucellosis & $10(6.1)$ & $9(7.4)$ & 0 & $1(9.1)$ \\
\hline Endocarditis & $3(1.8)$ & $3(2.5)$ & 0 & 0 \\
\hline
\end{tabular}

* Percentage calculated based on no. of male patients in each category.

species. When the patients were evaluated according to the duration of their symptoms, Brucella species growth was identified in $63(64.3 \%)$ of the 98 patients with acute brucellosis, 4 (30.8\%) of the 13 patients with subacute brucellosis and 1 (25.0\%) of the four patients with chronic brucellosis. One patient had Brucella species growth in his synovial fluid, but not in his blood culture.
Although the SAT results for brucellosis of 11 patients with growth in their blood cultures were below a 1:160 titre, the SAT results of those who had growth in their synovial fluids but not their blood cultures were positive, at a 1:40 titre. The SAT results for brucellosis of the two patients who had growth in their cerebrospinal fluids (CSFs), but not their blood cultures, were 
positive at 1:20 and 1:80 titres. Ten patients with neurobrucellosis were included in our study. In 4 (40\%) of these ten patients, the Brucella species was isolated from the patients' CSFs. The other six patients were diagnosed with neurobrucellosis using serology from their CSFs.

Among the 118 patients who were examined within the first month of treatment, $79(66.9 \%)$ responded to the administered treatment. Of the 67 patients who were followed up for observation, relapse occurred in 19 (28.4\%) patients. The relapse rate was $11.6 \%$ when all 164 patients were considered. Among the 19 patients who relapsed, 17 (89.5\%) were diagnosed with acute brucellosis and 2 (10.5\%) with chronic brucellosis. Focal involvement was present at the first diagnosis of 12 (63.2\%) of these patients; 11 patients received treatment for osteoarticular involvement and one received treatment for endocarditis. One of the patients who relapsed was diagnosed with infective endocarditis, while another had her joint prosthesis removed. The growth of the Brucella species was observed in the blood cultures of two patients. Although the SAT for brucellosis was negative in one patient during relapse, the results were positive among the other relapsed patients: a 1:1,280 titre in two patients, $1: 640$ titre in one patient, $1: 320$ titre in four patients, $1: 160$ titre in five patients, 1:80 titre in four patients and 1:40 titre in two patients. Titre increases (in comparison to post-treatment values) were identified in six of the relapsed patients.

Various side effects associated with the antibiotics used were observed in 72 (43.9\%) of the 164 patients. Some of these patients experienced multiple side effects. The most commonly observed side effect was doxycycline-induced nausea ( $\mathrm{n}=38$, $23.2 \%)$, followed by rashes $(n=18,11.0 \%$ ), elevated liver

Table V. Laboratory test results of the patients at the time of diagnosis.

\begin{tabular}{lc}
\hline Variable & Median (range) \\
\hline Sedimentation rate $(\mathrm{mm} / \mathrm{h})$ & $30(0-106)$ \\
C-reactive protein $(\mathrm{mg} / \mathrm{dL})$ & $1.6(0-19.2)$ \\
White blood cell count $\left(/ \mathrm{mm}^{3}\right)$ & $6,600(2,000-14,900)$ \\
Haemoglobin $(\mathrm{g} / \mathrm{dL})$ & $12.7(6.5-17.9)$ \\
Platelet count* $\left(/ \mathrm{mm}^{3}\right)$ & $259,061 \pm 100,641$ \\
Aspartate transaminase $(\mathrm{IU} / \mathrm{L})$ & $26(7-462)$ \\
Alanine transaminase $(\mathrm{IU} / \mathrm{L})$ & $32(7-762)$ \\
Positive Rose Bengal test & 99.3 \\
\hline
\end{tabular}

* Data presented as mean \pm standard deviation. ${ }^{+}$Data presented as \%. enzymes ( $n=14,8.5 \%)$, dizziness ( $n=8,4.9 \%)$ and disturbances in balance $(n=4,2.4 \%)$. No mortality was identified in the study. One patient had a paravertebral abscess and suffered from external sphincter incontinence as a sequela of abscess drainage.

\section{DISCUSSION}

As the symptoms observed in brucellosis are not specific, patient history (e.g. the consumption of unpasteurised milk and dairy products, and/or animal husbandry activities) plays a very important role in its diagnosis. ${ }^{\left({ }^{8}\right)}$ However, patient history cannot help to distinguish infected from non-infected persons. While the majority of the patients diagnosed with brucellosis in the present study had a history of consuming unpasteurised milk and dairy products, and/or animal husbandry activities, 48 (29.3\%) of the 164 patients did not report any risk factors, thereby raising the suspicion that supermarket products may have been prepared in inappropriate conditions. ${ }^{(9)}$ We hypothesise that contaminated market products may have been the main transmission route in these patients.

Brucellosis typically causes fever, fatigue, irritability, headaches and depressive symptoms. Physical examination of patients with brucellosis often reveals the presence of fever, hepatomegaly and splenomegaly. The complaints and physical examination findings at admission in other studies of patients diagnosed with brucellosis were similar to those observed in the present study. ${ }^{(10-18)}$ The most frequent complaints among subacute and chronic brucellosis patients were back and joint pain. We believe that spondylodiscitis should be investigated in patients with back pain.

In brucellosis, the patient's leucocyte count is generally normal or low at the time of diagnosis, while the erythrocyte sedimentation rate is usually variable. Haematological disorders such as anaemia, leucopenia, thrombocytopenia and clotting abnormalities may be observed.(1) In the present study, the leucocyte count among the 164 patients varied between $2,000 / \mathrm{mm}^{3}$ and $14,900 / \mathrm{mm}^{3}$, and higher leucocyte counts were observed among the patients with focal involvement. This finding supports studies in the literature asserting that focal involvement should be excluded in patients with leucocytosis. ${ }^{(19)}$ On the other hand, contrary to other studies, ${ }^{(20)}$ we found no statistically significant difference in terms of the C-reactive protein concentrations and sedimentation rates of patients with and without focal involvement. Given that the rate of liver

Table VI. Laboratory test results of the patients at the time of diagnosis, according to whether the patient had focal involvement.

\begin{tabular}{lcc}
\hline Variable & \multicolumn{2}{c}{ p-value } \\
\cline { 2 - 3 } & Focal involvement & No focal involvement \\
\hline White blood cell count $\left(10^{3} / \mathrm{mm}^{3}\right)$ & $6.8(3.7-14.9)$ & $5.6(2.0-12.4)$ \\
Haemoglobin $(\mathrm{g} / \mathrm{dL})$ & $12.7(9.3-17.9)$ & $12.6(6.5-16.0)$ \\
Platelet count $\left(10^{3} / \mathrm{mm}^{3}\right)$ & $261(44-621)$ & $222(53-595)$ \\
Aspartate transaminase $(\mathrm{IU} / \mathrm{L})$ & $23.5(8-427)$ & $32(7-462)$ \\
Alanine transaminase $(\mathrm{IU} / \mathrm{L})$ & $28(7-762)$ & $36(10-344)$ \\
C-reactive protein $(\mathrm{mg} / \mathrm{dL})$ & $2.4(0-19.2)$ & $3.8(0.3-17.9)$ \\
Sedimentation rate $(\mathrm{mm} / \mathrm{h})$ & $32.5(0-106)$ & $30(2-101)$ \\
\hline
\end{tabular}


involvement in brucellosis is high, high levels of AST and ALT cannot be overlooked. In the present study, AST level was found to be abnormally high in 41 (25.0\%) patients, while ALT level was found to be abnormally high in 57 (34.8\%) patients. Although Ertek et $\mathrm{al}^{(16)}$ observed a rate of liver involvement of $3.2 \%$, rates similar to those we observed (i.e. $34.8 \%$ ) have been reported in other studies. ${ }^{(11,13,18,21,22)}$

In the present study, 20 patients had a negative SAT for brucellosis, according to serological diagnostic criteria (titre $\geq 1: 160$ ), during the first admission. However, these patients were diagnosed with brucellosis using culture results or increasing titres. Thus, we believe that all diagnostic tools should be used in the attempt to diagnose brucellosis. In the present study, we found a high rate of positivity when using the Rose Bengal test. Although the specificity of this test was reported to be high in nonendemic areas, it was reported to be low in endemic areas. ${ }^{(10,23)}$ Therefore, we opine that the Rose Bengal test should not be used as the sole diagnostic tool in endemic areas and in patients who have a history of brucellosis. In such cases, the SAT for brucellosis should also be performed.

The osteoarticular system is the system that is most frequently involved in focal brucellosis, with a reported involvement frequency of $10 \%-80 \% .{ }^{(1,11,19)}$ Studies conducted in Turkey have shown that musculoskeletal involvement in brucellosis ranges from $19 \%-68 \%$. $^{(13,15,16,21)}$ The rate of osteoarticular involvement in the present study was $53.0 \%$. When evaluated based on the duration of the patients' symptoms, osteoarticular involvement was identified in $41.8 \%$ of the patients with acute brucellosis, $90.3 \%$ of the patients with subacute brucellosis and $72.7 \%$ of the patients with chronic brucellosis. Spondylodiscitis was identified in $73.6 \%$ of the patients with osteoarticular involvement. These high rates may be attributed to the fact that our centre is a tertiary care hospital; hence, patients who require complex care and those who are unresponsive to treatment are generally admitted to our centre.

Osteoarticular system findings include sacroiliitis, peripheral arthritis, spondylodiscitis, osteomyelitis and bursitis, although their prevalence varies greatly among studies. Some studies, such as those conducted by Pappas et al ${ }^{(11)}$ and Bosilkovski et al,(24 highlight that peripheral arthritis is the most common type of osteoarticular involvement, while other studies conducted in Turkey have found that sacroiliitisis is the most common. ${ }^{(16,21)} \mathrm{A}$ study conducted by Aydın et al ${ }^{(25)}$ showed that the most common osteoarticular involvement is peripheral arthritis, while a study by Özön et al ${ }^{(26)}$ showed that spondylodiscitis is more frequently observed. In a study conducted by Yüce et al,(14) the authors specified that the most frequent bone and joint involvements were spondylodiscitis and paravertebral abscesses (23.7\%), and that the rates of musculoskeletal system involvement varied in different studies due to the different imaging methods used. Similar to findings by Yüce et $\mathrm{al}^{\left({ }^{(14)}\right.}$ the most frequent musculoskeletal involvement among the patients in the present study was spondylodiscitis $(39.0 \%)$, followed by sacroiliitis (20.1\%). Peripheral arthritis was observed in 11 (6.7\%) patients, with one of the peripheral arthritis cases presenting in the form of polyarthritis. In the present study and other studies, ${ }^{(22,27,28)}$ peripheral arthritis was found to be most frequently identified in the knee joint.

Two main pathogenic mechanisms of peripheral arthritis have been suggested by Gotuzzo et al:(29) the septic and reactive forms. The synovial membrane is a highly vascular layer of connective tissue that lacks a basement membrane. ${ }^{(30)}$ This situation facilitates the entry of bacteria into the joint space during episodes of bacteraemia. ${ }^{(31)}$ It is possible that the synovial membrane's rich vascularity is the reason that the knee is the most commonly affected joint in peripheral arthritis.

This study was not without limitations. Firstly, it was a retrospective, single-centre study. There was also no long-term follow-up for all patients. Lastly, there was a lack of treatment evaluation for all patients within the first month of treatment.

Due to the prevalence of risk factors and the risk of chronicity, brucellosis remains a significant public health problem. It should be considered when patients living in areas that have high incidences of brucellosis present with high fever, joint pain and back pain. Given that the Rose Bengal test yielded a high rate of positivity among the patients with brucellosis in the present study, we suggest that this test be used in pre-diagnosis, even at sites where adequate laboratory facilities do not exist. The SAT for brucellosis should be performed in endemic regions. The results of the present study indicate that patients with symptoms for a prolonged period or who have inadequate or no response to brucellosis treatment should be examined for osteoarticular involvement using appropriate imaging methods. In addition, considering the frequency of relapse among the patients with focal involvement, brucellosis patients who present with high leucocyte counts should be examined for focal involvement using the required clinical examinations and radiography. Spondylodiscitis should also be excluded in brucellosis patients who suffer from continued back pain despite receiving appropriate treatment.

\section{REFERENCES}

1. Young EJ. Brucella Species. In: Mandell GL, Bennet JE, Dolin R, eds. Mandell, Douglas, and Bennett's. Principles and Practice of Infectious Diseases. 7th ed. Philadelphia: Churchill Livingstone Elsevier, 2010: 2921-5.

2. Yüce A, Alp-Çavuş S. [Brucellosis in Turkey: a review]. Klimik Derg 2006; 19:87-97. Turkish.

3. Çalık S, Gökengin AD. Human brucellosis in Turkey: a review of the literature between 1990 and 2009. Turk J Med Sci 2011; 41:549-55.

4. Gedikoğlu S, Helvaci S, Ozakin C, Gökirmak F, Kiliçturgay K. Detection of Brucella melitensis by BACTEC NR 730 and BACTEC 9120 Systems. Eur J Epidemiol 1996; 12:649-50.

5. Joint FAO/WHO Expert Committee on Brucellosis. Sixth report. Technical report series no. 740. Geneva: World Health Organization, 1986.

6. Taşova Y, Saltoğlu N, Yılmaz G, İnal S. [Brucellosis: investigation of clinical, laboratory and treatment properties of 238 adult cases]. Infeksiyon Dergisi 1998; 12:307-12. Turkish.

7. Colmenero JD, Requera JM, Fernández-Nebro A, Cabrera-Franquelo F. Osteoarticular complications of brucellosis. Ann Rheum Dis 1991; 50:23-6.

8. Rubach MP, Halliday JE, Cleaveland S, Crump JA. Brucellosis in lowincome and middle-income countries. Curr Opin Infect Dis 2013; 26:404-12.

9. Yumuk Z, O'Callaghan D. Brucellosis in Turkey--an overview. Int J Infect Dis 2012; 16:e228-35

10. Ruiz-Mesa JD, Sánchez-Gonzalez J, Reguera JM, et al. Rose Bengal test: diagnostic yield and use for the rapid diagnosis of human brucellosis 
in emergency departments in endemic areas. Clin Microbiol Infect 2005; 11:221-5

11. Pappas G, Akritidis N, Bosilkovski M, Tsianos E. Brucellosis. N Engl J Med 2005; 352:2325-36.

12. Memish Z, Mah MW, Al Mahmoud S, Al Shaalan M, Khan MY. Brucella bacteraemia: clinical and laboratory observations in 160 patients. J Infect 2000; 40:59-63.

13. Buzgan $\mathrm{T}$, Karahocagil MK, Irmak $\mathrm{H}$, et al. Clinical manifestations and complications in 1028 cases of brucellosis: a retrospective evaluation and review of the literature. Int J Infect Dis 2010; 14:e469-78.

14. Yüce A, Alp-Çavuş S, Yapar N, Çakır N. [Brucellosis: an evaluation of 55 cases]. Klimik Derg 2006; 19:13-7. Turkish.

15. Kayaaslan B, Akıncı E, Baştuğ A, et al. Analysis of 161 adult patients with brucellosis. Turk J Med Sci 2013; 43:187-93.

16. Ertek M, Yazgı H, Kadanalı A, Özden K, Taşyaran MA. Complications of Brucella infection among adults: an 18 year retrospective evaluation. Turk J Med Sci 2006; 36:377-81.

17. Eales KM, Norton RE, Ketheesan N. Brucellosis in northern Australia. Am J Trop Med Hyg 2010; 83:876-8.

18. Sathyanarayanan V, Razak A, Saravu K, et al. Clinical profile of brucellosis from a tertiary care center in southern India. Asian Pac J Trop Med 2011; 4:397-400.

19. Franco MP, Mulder M, Gilman RH, Smits HL. Human brucellosis. Lancet Infect Dis 2007; 7:775-86.

20. Kurtaran B, Candevir A, İnal AS, et al. Clinical appearance of brucellosis in adults: fourteen years of experience. Turk J Med Sci 2012; 42:497-505.

21. Aygen B, Doganay M, Sümerkan B, Yıldız O, Kayabaş Ü. Clinical manifestations, complications and treatment of brucellosis: a retrospective evaluation of 480 patients. Méd Mal Infect 2002; 32:485-93.
22. Hasanjani Roushan MR, Mohrez M, Smailnejad Gangi SM, Soleimani Amiri MJ, Hajiahmadi M. Epidemiological features and clinical manifestations in 469 adult patients with brucellosis in Babol, Northern Iran. Epidemiol Infect 2004; 132:1109-14.

23. Serra J, Viñas M. Laboratory diagnosis of brucellosis in a rural endemic area in northeastern Spain. Int Microbiol 2004; 7:53-8.

24. Bosilkovski M, Krteva L, Caparoska S, Dimzova M. Osteoarticular involvement in brucellosis: study of 196 cases in the Republic of Macedonia. Croat Med J 2004; 45:727-33.

25. Aydın G, Keleş I, Atalar E. [Clinical and laboratory features of patients with musculoskeletal brucellosis]. Turkiye Klinikleri J Med Sci 2005; 25:354-63. Turkish.

26. Özön A, Aydemir H, Pişkin N, et al. [Comparison of spondylitis and sacroileitis due to brucellar infection]. Klimik Derg 2005; 18:99-102. Turkish.

27. Hashemi SH, Keramat F, Ranjbar M, et al. Osteoarticular complications of brucellosis in Hamedan, an endemic area in the west of Iran. Int J Infect Dis 2007; 11:496-500.

28. Khateeb MI, Araj GF, Majeed SA, Lulu AR. Brucella arthritis: a study of 96 cases in Kuwait. Ann Rheum Dis 1990; 49:994-8.

29. Gotuzzo E, Alarcón GS, Bocanegra TS, et al. Articular involvement in human brucellosis: a retrospective analysis of 304 cases. Semin Arthritis Rheum 1982; 12:245-55.

30. Frank G, Eppes SC. Bone, joint, and soft tissue infections. In: Zaoutis LB, Chiang WV, eds. Comprehensive Pediatric Hospital Medicine, Philadelphia: Mosby, 2007: 414

31. Krogstad P. Septic arthritis. In: Cherry JD, Harrison GJ, Kaplan SL, et al, eds. Feigin and Cherry's Textbook of Pediatric Infectious Diseases, 7th ed, Philadelphia: Elsevier Saunders, 2014: 727. 